\title{
Correction to: Oxidative Stress and Immunological Complexities in Multidrug-Resistant Tuberculosis
}

Ridhima Wadhwa, Nikita Sehgal, Naresh G, Taru Aggarwal, Saurabh Satija, Meenu Mehta, Gaurav Gupta, Dinesh K. Chellappan, Murtaza M. Tambuwala, Brain Oliver, Trudi Collet, Pawan K. Maurya, Philip M. Hansbro, and Kamal Dua

\section{Correction to: \\ Chapter 7 in: P. K. Maurya, K. Dua (eds.), Role of Oxidative Stress in Pathophysiology of Diseases, https://doi.org/10.1007/978-981-15-1568-2_7}

The online version of the book was inadvertently published with an error in the last name of one of the authors in Chapter 7 as Murtaza M. Chellappan. The name has been corrected as Murtaza M. Tambuwala. 